\title{
Determinantes externos e internos da reforma curricular do curso de medicina da Pontifícia Universidade Católica de São Paulo
}

\author{
External and internal determinants of the curriculum reform of the \\ school of medicine of the Pontifícia Universidade Católica de São Paulo
}

Maria Valéria Pavan ${ }^{1}$, Maria Helena Senger ${ }^{1}$, Waldemar Marques²

\begin{abstract}
RESUMO
Introdução: Em 2006, o curso de Medicina da Faculdade de Ciências Médicas e da Saúde (FCMS) da Pontifícia Universidade Católica de São Paulo (PUC-SP) alterou seu projeto pedagógico, em prática desde a década de 1970, para utilizar métodos ativos de ensino/aprendizagem, ancorados na aprendizagem baseada em problemas. Como esperado, em uma instituição de ensino como a PUC-SP, que traz na história uma grande valorização do processo democrático, mudanças como essas não são impostas à comunidade acadêmica, mas se inserem em um contexto de ampla discussão, norteada por acontecimentos externos e internos à instituição. Objetivos: Identificar e analisar os principais determinantes externos e internos da reforma curricular de 2006. Métodos: Foram utilizadas pesquisa bibliográfica e consulta aos arquivos da FCMS da PUC-SP buscando identificar e contextualizar os movimentos sociais, institucionais e de órgãos governamentais da educação e da saúde que pretendiam mudanças curriculares nos cursos de Medicina no Brasil. Para entender os determinantes internos, buscou-se reconstruir o ambiente acadêmico no período da reforma, identificando os motivadores próprios da comunidade acadêmica. Resultados: A mudança do currículo do curso de Medicina da PUC-SP ocorreu dentro de um movimento externo amplo, voltado para a formação do profissional para além da especialização. Somou-se a isso um ambiente de insatisfação dos docentes com o modelo de ensino tradicional em prática. Conclusão: Optou-se por um modelo curricular adequado aos princípios da instituição, que incorporou as orientações das diretrizes curriculares nacionais com o compromisso de formar médicos comprometidos com o sistema de saúde do país.

Palavras-chave: educação médica; currículo; aprendizagem baseada em problemas; docentes.
\end{abstract}

\begin{abstract}
Introduction: In 2006, the medical school of the Faculdade de Ciências Médicas e da Saúde (FCMS) of the Pontifícia Universidade Católica de São Paulo (PUC-SP), changed its pedagogical project, in use since the 1970s, to use active teaching/learning methods, anchored in the Problem Based Learning. As expected, in an educational institution such as FCMS of PUC-SP, which brings a great appreciation of the democratic process, changes such as these are not imposed on the academic community, but are inserted in a context of broad discussion, guided by external and internal events to the Institution. Objectives: To identify and analyze the main external and internal determinants of the 2006 curricular reform. Methods: The bibliographic data and the researches in the FCMS of PUC-SP archives were used to understand and contextualize the social, institutional and governmental movements in promoting curricular changes in medical courses in Brazil. To understand the internal determinants, we sought to reconstruct the academic environment in the period of reform, identifying the motivators of the academic community. Results: The change in the curriculum of the medical course of the PUC-SP occurred within a broad external movement, aimed to graduate general practitioners beyond the specialization. Added to this, an environment of some teachers' dissatisfaction with the traditional pedagogical model in practice. Conclusion: It was opted for a curricular model appropriate to the institution's principles, which incorporated the national curricular guidelines for medical schools and with a commitment to graduate doctors committed to the country's health system.
\end{abstract}

Keywords: education, medical; curriculum; problem-based learning; faculty.

'Pontifícia Universidade Católica de São Paulo (PUC-SP), Faculdade de Ciências Médicas e da Saúde (FCMS) - Sorocaba (SP), Brasil.

${ }^{2}$ Universidade de Sorocaba (UNISO) - Sorocaba (SP), Brasil.

Contato:mvpavan@globo.com

Recebido em 04/03/2017. Aceito para publicação em 22/03/2017. 


\section{INTRODUÇÃO}

Hoje, no Brasil, a educação médica convive com dois modelos principais de ensino. Um modelo é chamado de tradicional, oriundo e difundido desde o início do século XX, a partir do Relatório Flexner, nos Estados Unidos da América (EUA). ${ }^{1,2}$ Esse modelo tem sido usado no Brasil desde meados do século XX e continua em prática na maior parte dos cursos de Medicina do país, mesmo nas instituições de ensino superior (IES) mais tradicionais e bem-conceituadas. É visto como um método que se utiliza da transmissão do conhecimento, no qual o aluno é passivo diante de um conteúdo de ensino fechado, previamente estabelecido pelo docente, fragmentado e baseado nas especialidades médicas. Além disso, é um modelo de treinamento de práticas médicas realizado preferencialmente em hospitais, com o estudo centrado na doença e em seu tratamento. ${ }^{1,3,4} \mathrm{O}$ outro modelo, nomeado como metodologias ativas de ensino e aprendizagem, ancorado na Aprendizagem Baseada em Problemas (ABP), surgiu no final da década de 1960, é conhecido como inovador, está centrado no estudante como agente de construção do seu conhecimento e voltado para a integração dos conteúdos das ciências básicas e clínicas, para a inserção do estudante em práticas médicas desde o início do curso e para a aproximação do aluno às reais necessidades da população. ${ }^{1,5}$ Esse modelo tem sido preferido pelas novas escolas médicas no Brasil e orientado pelas últimas diretrizes para os cursos de medicina. ${ }^{3,5,6}$

$\mathrm{Na}$ sua fundação, em 1949, a Faculdade de Medicina de Sorocaba, hoje Faculdade de Ciências Médicas e da Saúde (FCMS) da Pontifícia Universidade Católica de São Paulo (PUC-SP), seguiu o modelo curricular baseado na cátedra. A partir da década de 1970, quando foi incorporada pela PUC-SP, a FCMS da PUC-SP passou a seguir o modelo proposto pelo relatório de Flexner, ${ }^{1,2}$ orientando-se pelo Estatuto da Universidade. Esse modelo foi utilizado até o final de 2005, quando um novo projeto pedagógico foi apresentado à comunidade acadêmica, aprovado e implementado na turma ingressante de 2006, estando em vigência até os dias atuais. ${ }^{7,8}$ Desde então, o modelo curricular em prática no curso de Medicina da PUC-SP utiliza-se de quatro estratégias de ensino/aprendizagem, sendo elas a ABP, a Problematização, a Aprendizagem Baseada na Pesquisa ou por Projetos e a Aprendizagem Baseada na Prática. ${ }^{8}$

A mudança do projeto pedagógico de um curso que integra uma IES como a PUC-SP, que tem na sua história uma grande valorização do processo democrático, dentro e fora da instituição, não ocorreu de maneira imposta e, sim, fez parte da resposta a uma série de acontecimentos externos e internos à instituição. Neste sentido, o objetivo deste estudo foi identificar e analisar os principais determinantes externos e internos da reforma curricular, implementada a partir de 2006, no curso de Medicina da PUC-SP.

\section{MÉTODOS}

Para identificar os possíveis determinantes externos e internos da reforma curricular do curso de Medicina da FCMS da PUC-SP, a pesquisa foi direcionada, buscando entender e contextualizar temporalmente os movimentos e as intenções de órgãos governamentais das áreas da educação e da saúde que levaram às mudanças curriculares nos cursos da área da saúde, em especial da Medicina, a partir do final do século $\mathrm{XX}$. Para isso, foram utilizadas a pesquisa bibliográfica e a consulta a documentos disponíveis na FCMS da PUC-SP.

Para a análise dos determinantes externos, foi feita uma revisão histórica que, como descrito por Luna, ${ }^{9}$ pudesse ajudar a entender a inserção de determinados fatores dentro do assunto em estudo, a educação médica, e suas implicações nas mudanças ocorridas. Assim, foi traçado um paralelo entre as orientações da Constituição do Brasil de 1988, as orientações do Ministério da Saúde e da Educação, as conquistas dos movimentos sociais, as iniciativas governamentais por meio de apoios financeiros disponibilizados no período e o conteúdo do Projeto Pedagógico do Curso de Medicina da FCMS da PUC-SP em prática desde 2006, analisando o que este contemplava dessas orientações. Tomou-se como ponto de partida a Constituição do Brasil de $1988,{ }^{10}$ passando pela Lei $n^{\circ} 8.080$ de $1990,{ }^{11}$ que regulamenta o Sistema Único de Saúde (SUS), pela ata das Diretrizes Curriculares Nacionais do Curso de Graduação em Medicina de 20016 e pelas Diretrizes do Programa de Incentivo a Mudanças Curriculares. ${ }^{12}$ Ainda foi considerado como um fator externo o Projeto Pedagógico Institucional da PUC-SP. ${ }^{13}$

Para a análise dos determinantes internos, foi realizada uma análise documental, considerando documentos "[...] quaisquer materiais escritos que possam ser usados como fonte de informação sobre o comportamento humano" ${ }^{14} \mathrm{e}$ permita "[...] identificar informações factuais nos documentos a partir de questões ou hipóteses de interesse". ${ }^{14}$ Para isso, foram feitas buscas nos arquivos da FCMS da PUC-SP, tentando identificar evidências da participação da comunidade acadêmica nos movimentos sociais, políticos e governamentais identificados na pesquisa dos determinantes externos.

O projeto de pesquisa foi aprovado pelo Comitê de Ética em Pesquisa da Universidade de Sorocaba e foram obtidas autorizações da Coordenação do Curso de Medicina e da Direção da FCMS da PUC-SP para a realização da pesquisa.

O estudo foi realizado com financiamento dos pesquisadores.

\section{RESULTADOS}

\section{Determinantes externos da reforma curricular}

Entre os determinantes externos que contribuíram para a criação de uma nova proposta para o curso de Medicina da FCMS da PUC-SP estão:

- $\quad$ A Constituição de 1988 que traz pela primeira vez a saúde como um direito do cidadão e um dever do Estado, oferecendo um amplo espaço de discussão de demandas sociais e o elemento legal indispensável para a criação de um sistema de saúde universal para o país. ${ }^{10}$

- A concepção e implantação do SUS, nascido dos movimentos sociais e tendo como ponto central a garantia, pela Constituição de 1988, da saúde como direito a todos, que inclui em suas disposições de regulamentação 
a responsabilidade não só do Estado, mas também das pessoas, da família, das empresas e da sociedade para o seu bom funcionamento. ${ }^{11}$

- $\quad$ O resultado da avaliação das condições de oferta dos cursos de medicina pelo Departamento de Política do Ensino Superior, da Secretaria de Educação Superior do Ministério da Educação de 1999 que classificou as condições da instituição como "regulares", de maneira geral. ${ }^{8,15,16}$ As questões levantadas nessa avaliação, referentes à organização curricular, à organização didático-pedagógica, ao corpo docente e às instalações estavam em consonância com as manifestações que vinham sendo feitas pelos membros mais ativos da comunidade acadêmica local e puderam ser utilizadas como reforço à necessidade de revisão na forma de ensinar, buscando atingir níveis de excelência, dentro da classificação de qualidade de ensino.

- A promulgação, pelo Ministério da Educação (MEC), das Diretrizes Curriculares Nacionais do Curso de Graduação em Medicina, em 7 de novembro de $2001,{ }^{6}$ que orientavam para a organização dos cursos de Medicina, estimulando a formação do médico comprometido com as demandas do SUS.

- O lançamento, em 2002, pelo Ministério da Saúde e pelo MEC, do Programa de Incentivos às Mudanças Curriculares dos Cursos de Medicina (PROMED). ${ }^{12}$ Esse programa forneceu aporte financeiro aos cursos de Medicina que se dispuseram a adequar a formação profissional às diretrizes curriculares e às necessidades do SUS e possibilitou que a Comissão de Reforma Curricular do Curso de Medicina da PUC-SP, já organizada e em busca de meios para fazer a desejada mudança, elaborasse o projeto de reforma curricular que foi selecionado entre os dez melhores pelo PROMED.

- $\quad$ A aprovação, em 2004, do Projeto Pedagógico Institucional da PUC-SP com as orientações a todos os cursos de graduação "para a reformulação dos cursos de graduação no processo de implantação das novas diretrizes curriculares", ${ }^{13}$ ampliando o espaço de discussão do novo projeto da FCMS dentro da universidade, pois permitia a organização curricular em conteúdos disciplinares ou interdisciplinares (organizado em disciplinas, módulos, núcleos e outros); atividades práticas; estágios; atividades acadêmicas complementares (científicas e culturais); monitoria; iniciação científica; atividades de extensão como cursos e estágios de iniciativa do aluno; Trabalho de Conclusão de Curso (TCC) e outros componentes.

\section{Determinantes internos da reforma curricular}

- Seguindo as orientações do Programa de Integração Docente Assistencial (IDA), ${ }^{17}$ um marco do início do movimento da formação médica para além dos limites do hospital, extramuros, que ocorreu na década de 1980, no curso de Medicina da PUC-SP foi construído um projeto que buscava:
[...] a conjugação de atividade de ensino e pesquisa da PUC-SP através do Centro de Ciências Médicas e Biológicas, campus Sorocaba, com as atividades assistenciais do Serviço de Saúde na área de abrangência do Município de Sorocaba e um Campus Avançado de Tapiraí - SP. ${ }^{18}$

Embora esse projeto não tenha se consolidado, assim como aconteceu em muitas outras instituições de ensino médico na época, ele mostra que já havia, entre os membros da comunidade acadêmica, aqueles que acreditavam na necessidade de sair do modelo tradicional de ensino da Medicina, centrado no hospital.

- A avaliação do curso de Medicina da PUC-SP, no início da década de 1990, realizada pelos próprios docentes da faculdade, com o objetivo da reformulação do currículo, que identificou o conflito dos docentes entre duas vertentes:

[...] um grupo valorizava a formação do médico generalista [...] priorizavam as grandes áreas da medicina como Clínica Médica, Clínica Cirúrgica, Pediatria, Obstetrícia [...], enfatizaram a capacidade do médico em diagnosticar [...] valorizavam a formação humanística [...] Um grupo que acreditava mais na especialidade [...] o homem como ser essencialmente biológico e seus órgãos e aparelhos como identidades mais ou menos isoladas [...] a doença como desvio de caráter individual de uma norma padrão demonstrável por métodos técnicos científicos. ${ }^{19}$

A partir dessa avaliação, elaborou-se um projeto de aproximação do estudante de medicina às comunidades de baixa renda do município de Sorocaba. Mais tarde, esse projeto foi incorporado à matriz curricular, sendo, talvez, um dos primeiros movimentos de "saída dos muros" da FCMS da PUC-SP.

- A diminuição do poder dos núcleos docentes que tentavam manter sua força política baseada no Professor Catedrático ocorreu mediante o afastamento natural, ou imposto pela instituição, de grande parte dos docentes antigamente intitulados "chefes de cadeira", a partir do final da década de 1980. Embora a estrutura administrativa da FCMS da PUC-SP já fosse departamental desde a década de 1970, quando foi incorporada pela PUC-SP, que em seu Estatuto de 11 de fevereiro de 1969 organizou a estrutura administrativa e didática da instituição seguindo as orientações da Reforma Universitária de $1968,{ }^{17,20}$ houve dificuldade na aceitação das mudanças de conceitos que se seguiam a isso, onde se inseria a coordenação das disciplinas e resoluções por meio do diálogo entre os pares e não pelo autoritarismo. A dificuldade no enfrentamento diante desse núcleo de poder pode ser avaliada quando se constata que, ainda hoje, há quem utilize o termo chefe de disciplina.

- $\quad$ A participação da FCMS, em 1996, na primeira fase do projeto Comissão Interinstitucional de Avaliação do Ensino Médico (CINAEM), por meio dos seus alunos, 
que se submeteram aos testes de avaliação cognitiva no início e no final do sexto ano do curso de Medicina, e a participação de alguns docentes nas reuniões da segunda fase do projeto CINAEM. ${ }^{21,22}$ Lembrando que a Associação Médica Brasileira (AMB), em 1991, criou a CINAEM, uma comissão que agregou entidades médicas de todo país. Essa comissão assumiu, em nível nacional, o papel de articular técnica e politicamente um movimento a favor da reforma do ensino médico e elaborar um projeto para avaliação do ensino médico no Brasil. Embora o projeto CINAEM não tenha conseguido atingir todas as metas propostas, ele foi fundamental para a elaboração das Diretrizes Curriculares Nacionais do Curso de Graduação em Medicina de 2001. . $^{61}$

- A percepção dos departamentos e da Coordenação do Curso de Medicina, a partir da década de 1990, da necessidade de revisão do modelo disciplinar, da urgência da aproximação entre as disciplinas básicas e as clínicas, da inadequação da matriz curricular que apresentava grande distância entre o ensino teórico e prático, da necessidade de grupos menores de alunos nas atividades de aprendizagem, da falta de apoio pedagógico para o ensino e da necessidade de qualificação do corpo docente. Além disso, já se apontava para a necessidade de uma reforma curricular que estimulasse o aluno a procurar o conhecimento e para a necessidade de melhorias estruturais no campus Sorocaba da PUC-SP.

[...] através de um novo desenho curricular não se modifique apenas a grade horária, mas que se busque um novo enfoque cultural para o médico que se vai formar [...] criar uma mentalidade no alunato de estudo constante [...] É fundamental que se consiga uma assessoria pedagógica presente no campus Sorocaba, bem como mudanças estruturais e melhorias nas salas de aula e biblioteca. ${ }^{23}$

Dentre essas falhas, uma das mais graves tem sido a falta de integração entre os programas das disciplinas afins, o que tem dificultado se chegar a um objetivo comum. Se faz desta forma urgente a integração interdisciplinar e a sistematização de alguns procedimentos acadêmicos, com finalidade única de optimização de nossa infraestrutura e melhora na qualidade do ensino, diante da globalização acelerada que o conhecimento científico tem passado. ${ }^{24}$

- A manifestação de insatisfação dos alunos em relação à duração do internato, pedindo a sua ampliação. Em 2001, o internato ainda era de um ano no curso de Medicina da PUC-SP, enquanto em praticamente todos os outros cursos do estado de São Paulo era de 2 anos.

Observa-se uma vital importância desta aprovação, o que levará consequentemente a uma melhoria no aprendizado e formação acadêmica, ampliando nossas chances de conquista no amargo processo de seleção para o ensino de pós-graduação (Residência Médica), atendendo desta forma os critérios impostos pelas entidades que oferecem o curso de Residência Médica. ${ }^{25}$

- Novas iniciativas entre os docentes, com projetos de movimentação dos alunos para além dos muros da faculdade, como a criação do ambulatório escola, em uma unidade básica de saúde. ${ }^{26}$

- A percepção, pelos docentes, da falta de motivação dos alunos e da necessidade de mudanças no modelo de ensino.

\begin{abstract}
Meses antes de mudar o currículo, eu, sinceramente, tinha pensado em parar de dar aula, porque era um desgaste enorme. Eu dou aula desde 85. Eu sempre gostei. Acho uma coisa muito gratificante. Mas nos últimos anos do currículo velho eu estava desanimado, porque eu ia lá dar aula para 100 alunos e ninguém prestava atenção. Então eu sempre vi com bons olhos a ideia da mudança, porque eu não sabia o que fazer, mas eu sabia que uma mudança tinha que acontecer. ${ }^{16}$
\end{abstract}

\section{Considerações sobre a reforma}

Por meio da análise dos possíveis determinantes da reforma curricular, foi possível inferir que, no final da década de 1990 e início da década de 2000, a comunidade acadêmica da FCMS da PUC-SP sentia a necessidade da atualização e mudança no curso de Medicina. Ela não estava à margem do movimento de reformulação do ensino médico que já existia em algumas IES, mas não parecia, ainda, organizada para efetivar essa mudança.

Como bem observado por Feuerwerker, ${ }^{27}$ para que as reformas aconteçam deve haver uma sequência de situações favorecedoras envolvendo a comunidade acadêmica, a administração da instituição e disposição dos órgãos públicos administradores da saúde nos seus diferentes níveis, isto é, municipal, estadual e federal. Além da articulação política por parte daqueles que pretendem gerenciar o processo, é preciso haver consonância entre esses diversos setores e a proposta de reforma. Neste sentido, entre 2000 e 2008, docentes com experiência na carreira acadêmica e administrativa ocuparam, por duas gestões, cargos eletivos centrais na administração da FCMS da PUC-SP. Com isso, reunindo conhecimento das demandas da comunidade, disposição, poder de decisão e comprometimento com o processo de mudanças exigidas, eles foram os artífices da reforma.

A viabilização das mudanças também foi favorecida pelo reconhecimento dessa nova equipe pela reitoria da universidade, pela conquista do financiamento externo (PROMED) e pela capacidade de articulação política da comissão de reforma, estabelecendo parcerias nos novos cenários de práticas médicas, particularmente na atenção primária à saúde. Além disso, a comunidade acadêmica parece ter se sensibilizado em relação à necessidade de mudança, principalmente naquilo que se refere ao modelo de ensino/aprendizagem. 
A decisão de mudanças desse tipo pode vir de cima para baixo, da cúpula diretora da instituição, ou ao contrário, pode vir da base do grupo, de técnicos ou de especialistas, mas "de qualquer maneira, a responsabilidade de iniciar e dirigir uma mudança cabe à direção, que deve decidir e indicar o caminho a se tomar, o qual deve ser coerente com a visão institucional", ${ }_{28}$ "deve entrar pela porta da frente, deve ser compromisso do núcleo central do poder, ou seja, a direção". ${ }^{27}$

Neste caso em estudo, a direção da FCMS da PUC-SP tomou para si a responsabilidade de articular as mudanças necessárias para fazer a reforma e os seus protagonistas foram os mesmos docentes que discutiam a necessidade de mudanças nos anos anteriores. Assim, a reforma aconteceu no momento em que os membros da direção acreditavam na necessidade de se fazer alguma mudança para reativar o entusiasmo dos docentes, ou seja, quando os "mudancistas" chegaram ao poder. ${ }^{27}$

\section{CONSIDERAÇÕES FINAIS}

A mudança do currículo do curso de Medicina da PUC-SP ocorreu no interior de um movimento mais amplo que enfatiza a formação de um profissional para além da especialização e que se integra a um contexto e conceito mais amplo de saúde enquanto dimensão social.

A esse contexto externo se somou um ambiente institucional de insatisfação com o curso tal como vinha sendo desenvolvido, no qual as possibilidades de mudanças estavam também vinculadas à disponibilidade de recursos financeiros para capacitação dos docentes, aquisição de equipamentos e adequações da estrutura física.

A inclusão da FCMS da PUC-SP dentro das escolas médicas contempladas com os recursos do PROMED foi muito importante para a viabilização da reforma, que dificilmente poderia acontecer sem aporte financeiro.

Optou-se por um modelo curricular adequado aos princípios da instituição, que incorporou as orientações das diretrizes curriculares nacionais e com compromisso de dar ao médico, formação para atender ao sistema de saúde do país.

\section{REFERÊNCIAS}

1. Baum KD, Axtell S. Trends in North American medical education. Keio J Med. 2005;54(1):22-8.

2. Flexner A. Medical education in the United States and Canada. New York: The Carnegie Foundation for the Advancement of Teaching; 1910. (Bulletin Number Four).

3. Lampert J. Dois séculos de Escolas Médicas no Brasil e a avaliação do ensino médico no panorama atual e perspectivas. Gaz Méd Bahia [Internet]. 2008 [acesso em 14 fev. 2017];78(1):31-7. Disponível em: http://www. gmbahia.ufba.br/index.php/gmbahia/article/view/255.

4. Santos SR. O aprendizado baseado em problemas (Problem-based learning - PBL). Rev Bras Educ Méd. 1994;18(3):121-4.

5. Berbel NAN. A problematização e a aprendizagem baseada em problemas: diferentes termos ou diferentes caminhos? Interface Comun Saúde Educ. 1998;2(2):139-54.
6. Brasil. Conselho Nacional de Educação. Câmara de Educação Superior. Resolução CNE/CES no 4/2001. Diretrizes Curriculares Nacionais do Curso de Graduação em Medicina [Internet]. 2001 [acesso em 14 fev. 2017]. Disponível em: http://portal.mec.gov.br/cne/ arquivos/pdf/CES04.pdf

7. Pavan MV, Senger MH, Marques W. Educação médica em foco. Rev Fac Ciênc Méd Sorocaba. 2013;15(2):39-43.

8. Pontifícia Universidade Católica de São Paulo. Proposta de alteração do projeto pedagógico do Curso de Medicina da Faculdade de Ciências Médicas e da Saúde, Campus Sorocaba. Sorocaba: PUC-SP; 2009.

9. Luna SV. A revisão de literatura como parte integrante do processo de formulação do problema. In: Luna SV. Planejamento de pesquisa: uma introdução. São Paulo: EDUC; 1996.

10. Brasil. Constituição Federal de 1988 [internet]. 1988 [acesso em 10 jan. 2016]. Disponível em: http://www.planalto.gov.br/ccivil_03/constituicao/ constituicaocompilado.htm

11. Brasil. Presidência da República. Lei $\mathrm{n}^{\circ}$ 8.080, de 19 de setembro de 1990. Dispõe sobre as condições para a promoção, proteção e recuperação da saúde, a organização e o funcionamento dos serviços correspondentes e dá outras providências. [internet]. 1990 [acesso em 12 fev. 2017]. Disponível em: http:// www.planalto.gov.br/ccivil_03/leis/18080.htm.

12. Brasil. Portaria interministerial $n^{\circ} 610$, de 26 de março de 2002. Projeto de incentivo a mudanças curriculares em cursos de medicina. Brasília: Ministério da Saúde; 2002.

13. Pontifícia Universidade Católica de São Paulo. Deliberação $n^{\circ}$ 11/2004. Projeto Pedagógico Institucional - PPI Diretrizes para a Graduação. São Paulo: PUC-SP; 2004.

14. Lüdke M, André MEDA. Pesquisa em educação: abordagens qualitativas. São Paulo: EPU; 1986. p. 39.

15. Brasil. Ministério da Educação. Secretaria de Educação Superior. Departamento de Política de Ensino Superior. Avaliação das condições de oferta dos cursos de medicina: 1999. Brasília: Ministério da Educação; 2000.

16. Pavan MV. A reforma curricular em um curso de medicina: determinantes externos e internos e o impacto sobre o trabalho docente [tese]. Sorocaba: Universidade de Sorocaba; 2016.

17. Marsiglia RG. Relação ensino/serviços: dez anos de integração docente assistencial (IDA) no Brasil. São Paulo: Hucitec; 1995.

18. Pontifícia Universidade Católica de São Paulo. Centro de Ciências Médicas e Biológicas. Projeto PIDA: ingresso na segunda etapa do programa de apoio à Educação Superior "Nova Universidade". Sorocaba: PUC-SP; 1986.

19. Cappelletti IF. Avaliação de currículo: limites e possibilidades. In: Cappelletti IF, editor. Avaliação, políticas e práticas educacionais. São Paulo: Articulação Universidade/Escola; 2002. p. 13-37. 
20. Martins CB. A reforma universitária de 1968 e a abertura para o ensino superior privado no Brasil. Educ Soc. 2009;30(106):15-35.

21. Piccini RX, Facchini LA, Santos RC. Transformando a educação médica brasileira: Projeto CINAEM: III Fase. Comissão Interinstitucional Nacional de Avaliação do Ensino Médico; 1998.

22. Pontifícia Universidade Católica de São Paulo. Centro de Ciências Médicas e Biológicas. Relatório sobre o desempenho cognitivo inicial e final: prova do CINAEM. Sorocaba: PUC-SP; 1998.

23. Pontifícia Universidade Católica de São Paulo. Departamento de Medicina. Planejamento acadêmico trienal: $2^{a}$ fase. Sorocaba: PUC-SP; 1997.

24. Pontifícia Universidade Católica de São Paulo. Centro de Ciências Médicas e Avaliação do Curso de Medicina. Sorocaba: PUC-SP; 1999.
25. Pontifícia Universidade Católica de São Paulo. Centro Acadêmico Vital Brasil. Abaixo assinado: interesse pela mudança da carga horária do internato, 2001. Sorocaba: Centro Acadêmico Vital Brasil; 2001.

26. Anjos RMP, Gianini RJ, Minari FC, Luca AHS, Rodrigues MP. "Vivendo o SUS": uma experiência prática no cenário da atenção básica. Rev Bras Educ Med. 2010;34(1):172-83.

27. Feuerwerker LCM. Além do discurso de mudança na educação médica: processos e resultados. São Paulo: Hucitec; 2002.

28. Moesby E. Perspectiva geral da introdução e implementação de um novo modelo educacional focado na aprendizagem baseada em projetos e problemas. In: Araújo UF, Satri G, editores. Aprendizagem baseada em problemas no ensino superior. São Paulo: Summus; 2009. p. 43-78. 